\title{
Potential Drug-Drug Interactions in Hospitalized Medical Patients: Data From Low Resource Settings
}

\author{
Khalid Rashid ${ }^{1}$, Yahya Khan ${ }^{2}$, Farrukh Ansar ${ }^{3}$, Aamir Waheed ${ }^{4}$, Muhammad Aizaz ${ }^{5}$ \\ 1. Internal Medicine, James Cook University Hospital, Middlesbrough, GBR 2. Department of Medical Education, Pak \\ International Medical College, Peshawar, PAK 3. Medicine, Northwest School of Medicine, Khyber Medical University, \\ Peshawar, PAK 4. Internal Medicine, Northampton General Hospital, Northampton, GBR 5. Community Health \\ Sciences, Peshawar Medical College, Peshawar, PAK
}

Corresponding author: Khalid Rashid, khalidrashid93@gmail.com

\section{Abstract \\ Introduction}

Adverse events related to Drug-Drug Interactions (DDIs) are among the few common reasons for hospitalization worldwide; however, they can be prevented with an efficient patient-centered system. Different mechanisms have successfully limited the prevalence of DDIs in developed countries. There are limited data regarding DDIs from limited-resource settings. Furthermore, there is no cost-effective system that has shown promising results in preventing them in this setting. This study aims to assess the frequency of potential DDIs in a low-resource setting and to check its association with different factors such as polypharmacy and demographics.

\section{Methods}

Through this cross-sectional study, drug charts of patients admitted to a medical unit in November 2019 were analyzed using a structured questionnaire. A list of drugs co-prescribed to each patient was entered into the Medscape Drug Interaction checker to calculate the frequency and severity of potential DDIs.

\section{Results}

The mean age of patients was 49 years, and on average, seven drugs were prescribed to each patient. Among 100 analyzed prescriptions, 400 potential DDIs were identified with a mean of $4 \pm 5.42$ per patient. According to Medscape interaction checker classification, 2 DDIs were contraindicated, 28 were serious, 246 required close monitoring, and 124 were minor. The most frequently encountered drug interaction was "spironolactone with furosemide." There was a significant correlation of the occurrence of potential DDIs with increased numbers of prescribed drugs.

Review began 07/27/2021 Review ended 08/16/2021 Published 08/20/2021

() Copyright 2021

Rashid et al. This is an open access article distributed under the terms of the Creative Commons Attribution License CC-BY 4.0., which permits unrestricted use, distribution, and reproduction in any medium, provided the original author and source are credited.

\section{Conclusion}

Our patient population was prescribed more drugs per patient than calculated in other settings. Polypharmacy is an independent risk factor for DDIs. Lastly, advancing age exposes patients to poly-pharmacy, and therefore, they are at a higher risk of developing DDIs.

Categories: Other, Therapeutics, Health Policy

Keywords: drug-drug interactions, polypharmacy, low resource setting, prescription audit, adverse outcomes

\section{Introduction}

Drug safety is an essential part of treatment and patient health, particularly in a hospital setting. A crucial aspect of drug safety is the drug-drug interactions (DDIs) that can lead to severe side effects and affect therapeutic efficacy. Adverse drug events (ADEs) are defined as injuries resulting from medical intervention related to a drug [1-3]. They are unwanted consequences of drug administration and are the leading cause of the increase in hospital stay and mortality worldwide, including in developed countries [2-4]. Most ADEs are due to interactions between different drugs, as most patients admitted to the hospital are on poly-pharmacy $[2,3]$. These DDIs have been commonly reported in high-risk populations such as the elderly and patients with multiple comorbidities. The main reason for this distribution is that the probability of poly-pharmacy increases with age, and also, most chronic diseases are treated with multidrug regimens [5]. Studies have also shown a deficiency in the prescription process and a need for more sensitivity in healthcare professionals regarding DDIs [5-7].

Developed countries have reported success in curtailing the incidence of DDIs by introducing various measures and initiatives. For instance, Halkin et al. reported a significant reduction in DDIs after introducing digital prescription methods and interaction screening across pharmacies and hospitals [6]. 
Garg et al. [7] have also reported a significant reduction in ADEs due to computerized clinical decision support systems (CDSSs). Other studies have also supported the role of interventions in preventing ADEs, including organizational modifications and educational measures [7-9].

Mousavi and Ghanbari [10] have reported a high prevalence of potential DDIs in the hospital setting in developing countries. Similar results have also been published by Ismail et al. [11] and others [12-15]. However, data that examine the prevalence of potential DDIs in a low-resource setting is very scarce. Therefore, a thorough study for evaluating potential DDIs is essential to assess the prevalence of potential and preventable drug interactions and risk factors associated with them. This would be helpful for designing and implementing efficient and cost-effective measures to prevent DDIs in these settings. This study aims to calculate the number of potential DDIs in the in-patient setting, and determining their risk factors.

\section{Materials And Methods}

Our study was a retrospective cross-sectional study conducted at a 125-bed tertiary teaching hospital with both medical and surgical in-patient facilities. Before the initiation of our study, formal written approval was taken from the Ethical Review Board (number: PRM/21/14376). Our study population included all the patients admitted consecutively to the Internal Medicine department from November 1, 2019 to November 30, 2019.

Prescription charts from medical records of all these patients were consulted after the permission of the hospital administration. A total number of 104 drug charts were analyzed, however, four were not included in the analysis as these patients were only prescribed one drug during their short stay. Therefore, data from 100 drug charts were included in the study population.

Patients demographics, including age and gender, were noted, and a list of drugs prescribed to every patient was generated. Subsequently, potential DDIs between co-prescribed drugs were checked using Medscape Drug Interaction Checker, and all the possible DDIs were identified. The potential DDIs were classified into four categories: contraindicated, avoid or use alternative/serious, monitor closely, and minor. The correlation of different factors with DDIs, including age, gender, and the total number of drugs prescribed, were analyzed using Statistical Package For the Social Sciences (SPSS) version 27.0 software (IBM Corp., Armonk, NY).

The objectives of this study were to calculate the frequency of potential DDIs through prescription analysis among the in-patient population of a teaching hospital and to determine the risk factors associated with these potential DDIs.

\section{Results}

Out of 100 enrolled patients in our study sample, 52 were male, and 48 were female. The mean age of patients was $49.52 \pm 17.5$, ranging from 13 to 84 . Age distribution categories are shown in Table 1 . The total number of drugs prescribed was 694, ranging between 2 and 15 per patient, with a mean of $6.93 \pm 2.94$. Moreover, $20 \%$ of the patients were prescribed 10 or more drugs on their prescription. Important baseline characteristics are shown in Table 1. Overall, 400 DDIs were identified in our study sample ranging between 0 and 30 per prescription, and the mean DDIs for each patient was $4 \pm 5.42$. At least one drug interaction was found in prescriptions of $77 \%$ of the patients. We identified one DDI in 23 prescriptions, two DDIs in 13 prescriptions, three and four DDIs in four and eight prescriptions, respectively, while $12 \%$ of the patients in our sample were exposed to 10 or more DDIs. As per the criteria used in our study, two DDIs fall in the category of contraindicated, 28 (serious), 246 (monitor closely), and 124 (minor) (Table 2). Our results revealed that the most common DDI was spironolactone with furosemide, whereas other interactions of furosemide, spironolactone, aspirin, esomeprazole with other drugs were frequent. The most common DDIs are reported in Table 3. 


\section{Cureus}

Characteristics

Frequency, N (\%)

Gender

Male

Female

Age (mean $\pm S D)$

$<20$

$21-40$

$41-60$

$61-80$

$81-100$

Prescribed drugs per patient

$<3$

$3-7$

$>7$

DDIs found in the number of patients

$1-3$

4-7

$>7$
$52(52)$

$48(48)$

$49.52 \pm 17.5$

$6(6)$

$26(26)$

$44(44)$

$21(21)$

3 (3)

$1(0.2)$

62 (45.3)

37 (54.3)

40 (40)

$22(22)$

$15(15)$

\section{TABLE 1: Baseline characteristics of patients}

\begin{tabular}{|c|c|c|}
\hline Severity of DDls & Number of DDIs & DDIs present in the number of prescriptions \\
\hline Contraindicated DDIs & 2 & 1 \\
\hline Serious DDIs & 28 & 17 \\
\hline Monitor closely DDls & 246 & 55 \\
\hline Minor DDls & 124 & 61 \\
\hline
\end{tabular}

TABLE 2: Severity of DDIs

DDI: drug-drug interactions. 


\section{Cureus}

\begin{tabular}{|c|c|c|c|}
\hline Interaction & Frequency & $\begin{array}{l}\text { Severity } \\
\text { (category) }\end{array}$ & Potential adverse outcome \\
\hline $\begin{array}{l}\text { Spironolactone with } \\
\text { furosemide }\end{array}$ & 20 & Monitor closely & Serum potassium imbalance \\
\hline Aspirin with furosemide & 8 & Monitor closely & Serum potassium imbalance and pharmacodynamic antagonism \\
\hline Aspirin with spironolactone & 8 & Monitor closely & Increase serum potassium \\
\hline Albuterol with furosemide & 6 & Monitor closely & Pharmacodynamic synergism and hypokalemia \\
\hline $\begin{array}{l}\text { Carvedilol with } \\
\text { spironolactone }\end{array}$ & 6 & Monitor closely & Increase serum potassium \\
\hline $\begin{array}{l}\text { Metoclopramide with } \\
\text { acetaminophen }\end{array}$ & 6 & Minor & Aspirin levels rise in blood \\
\hline $\begin{array}{l}\text { Metronidazole with } \\
\text { acetaminophen }\end{array}$ & 4 & Minor & Hepatic enzyme CYP2E1 metabolism affected \\
\hline $\begin{array}{l}\text { Esomeprazole with } \\
\text { cyanocobalamin }\end{array}$ & 4 & Minor & Decreases Gl absorption \\
\hline Rifampin with isoniazid & 3 & Serious & Risk of isoniazid toxicity \\
\hline $\begin{array}{l}\text { Esomeprazole with } \\
\text { clopidogrel }\end{array}$ & 2 & Serious & $\begin{array}{l}\text { Esomeprazole decreases the effects of clopidogrel by affecting hepatic enzyme } \\
\text { CYP2C19 metabolism }\end{array}$ \\
\hline $\begin{array}{l}\text { Ciprofloxacin with } \\
\text { theophylline }\end{array}$ & 2 & Serious & $\begin{array}{l}\text { Ciprofloxacin increases the effect of theophylline which may result in cardiac } \\
\text { arrest or seizure }\end{array}$ \\
\hline Linezolid with formoterol & 1 & Contraindicated & Risk of acute hypertensive episode \\
\hline Linezolid with albuterol & 1 & Contraindicated & Risk of acute hypertensive episode \\
\hline
\end{tabular}

TABLE 3: Important and common pairs of drug interactions

Correlation analysis showed that poly-pharmacy was significantly correlated with increased chances of DDIs with Pearson correlation $r=0.628$ and a $p$-value of $<0.001$. However, other correlation statistics were not significant ( $\mathrm{p}$-value $>0.05$ ). Multinomial regression analysis was done, which suggested that the male population was more prone to potential DDIs ( $\mathrm{OR}=3.92$, CI: $1.35-11.41$, $\mathrm{p}$-value $=0.012$ ). Similarly, patients prescribed more than seven drugs had higher odds of experiencing a DDI (OR=4.40 CI: 1.26-15.34, pvalue $=0.020$; Table 4 ). 


\section{Cureus}

\begin{tabular}{|c|c|c|c|c|}
\hline \multirow{2}{*}{ Variable } & \multicolumn{2}{|c|}{ Drug interactions } & \multirow{2}{*}{ P-value } & \multirow{2}{*}{ OR (95\% Cl) } \\
\hline & Present & Absent & & \\
\hline \multicolumn{5}{|l|}{ Gender } \\
\hline Male & 45 & 7 & 0.012 & $3.92(1.35-11.41)$ \\
\hline Female & 32 & 16 & & Reference \\
\hline \multicolumn{5}{|c|}{ Prescribed drugs per patient } \\
\hline $3-7$ & 44 & 16 & & Reference \\
\hline$>7$ & 33 & 4 & 0.020 & $4.40(1.26-15.34)$ \\
\hline \multicolumn{5}{|l|}{ Age } \\
\hline$<20$ & 4 & 2 & 0.144 & $0.202(0.024-1.72)$ \\
\hline $21-40$ & 20 & 6 & 0.420 & $0.535(0.117-2.44)$ \\
\hline $41-60$ & 33 & 11 & 0.451 & $0.595(0.154-2.29)$ \\
\hline $61-80$ & 20 & 4 & & Reference \\
\hline
\end{tabular}

\section{TABLE 4: Potential DDIs and their associations with the risk factors}

DDIs: drug-drug interactions.

\section{Discussion}

ADEs significantly increase patient morbidity and mortality and increases the length of hospital stay and healthcare costs. DDIs are one of the causative factors for ADEs, with Pirmohamed et al. reporting that an estimated $16.6 \%$ of all adverse drug reactions are caused by DDIs [16]. Drug interactions are preventable, but their adverse effects are among the primary reasons for hospital admission and associated complications.

The present study evaluated the presence of potential DDIs in the Internal Medicine ward of the index hospital. A total of 400 DDIs were identified; the mean DDIs for each patient was four, with at least one potential DDI in $77 \%$ of the patients (overall prevalence). This result is similar to a study done in Iran, which reported at least one potential DDI in $86.2 \%$ of the patients [10]. Another study from Pakistan reported at least one DDI in $52.8 \%$ of the study sample [11], while a study conducted in India found the prevalence of potential DDIs in admitted patients to be $30.67 \%$ [17]. Studies from the USA and Europe show a prevalence rate of $25 \%$ and $46 \%$, respectively $[18,19]$. The mean DDIs per patient were found to be four in the current study, which is lower than an Iranian study showing an average of 7.6 DDIs per patient [10]. However, our average number of DDIs was relatively higher than few other studies showing an average of 1.2 and 1.4 DDIs per patient $[20,21]$.

This comparison demonstrates that DDIs are common in various clinical settings and require immediate attention. However, every drug interaction is not fatal particularly in critical patients where a multi-drug regimen may be necessary, and few drugs interactions are unavoidable. However, careful monitoring of these patients is required. Therefore, identifying the severity of each DDI is necessary to evaluate the clinical significance and appropriate management. In the present study, the DDIs were divided into four categories; $0.5 \%$ of the DDIs were "Contraindicated," $7 \%$ were "Serious," $61.5 \%$ were in the "Monitor Closely" category, and $31 \%$ of the potential DDIs were "Minor." This shows that DDIs with serious adverse effects were less frequent in our study sample. This has also been shown by other studies, such as reported by Egger et al. [21] and others $[10,11]$.

Our analysis showed that a multidrug regimen was significantly associated with increased chances of DDIs. Different studies conducted worldwide bolster our evidence by reporting the same results [11,22]. Polypharmacy is a known factor for increased risk of potential DDIs [23]. Although there is no consensus on the definition of poly-pharmacy, the most commonly used definition is using five or more drugs daily [24]. Various studies have shown that potential DDIs are frequent when patients receive prescriptions with multiple drugs [23]. DDIs cause adverse effects and therapeutic inefficiency in many cases, with subsequent poor control of the ailment under treatment [18]. In our study, the mean number of drugs prescribed was 6.93. A study from Northwest Ethiopia reported a mean of 5.59 drugs per prescription [20], while a Brazilian study reported a mean of 5.6 per prescription [25]. The current study shows that poly-pharmacy was significantly correlated with increased chances of DDIs, as shown by other studies. 
As evident from our results, patients with advancing age are at greater risk of being prescribed a higher number of regular medications. Similar results have been shown by a study conducted in an almost identical setting by Ismail et al. [11].

As the population is getting older, more people with complex multisystem illnesses such as diabetes and hypertension are prescribed multiple drugs. Most of these drugs are for long-term use and require regular review by pharmacists or clinicians. This concept is often termed deprescribing [26], which is defined by Reeve et al. as the withdrawal process of an inappropriate medication, supervised by a healthcare professional to manage poly-pharmacy and improve outcomes [26]. This term also encompasses a reduction in the dose of the medication in addition to suspending the inappropriate medication [27].

However, this is a demanding task in settings with no proper follow-up. Moreover, in our settings, there is no concept of ward pharmacists reviewing patient's medication while they are inpatient. As a result, a significant number of patients end up taking unnecessary drugs. This practice puts them at risk of developing side effects of that drug and its potentially harmful interactions with other medications.

Reeve et al. analyzed the feasibility of a patient-centered deprescribing system for patients with unnecessary prescriptions of proton pump inhibitors (PPI). It was proved to be efficient in a small size sample of 57 [28]. Moreover, Moorehouse used a pilot model known as the Palliative and Therapeutic Harmonization (PATH) for making complex medical decisions for the older population based on their frailty assessment and cognition. PATH was shown to be effective in this study population of 200 [29].

There are other similar interventions designed to reduce polypharmacy-related adverse events in the elderly population. However, no standard universal tool can be implemented in all settings and is proven effective in large-scale settings. Therefore, keeping in mind the economic burden of this problem, an international body (International Group for Reducing Inappropriate Medication Use and Polypharmacy (IGRIMUP)) was formulated to slow down the adverse effects of this imminent epidemic. They have published their proposed strategies and recommendations to reduce inappropriate drug use and poly-pharmacy. Lastly, they have urged for an urgent and integrated approach to enlist inappropriate medication prescription as the leading global target of the highest priority [30].

\section{Limitations}

Due to a lack of sufficient local data, it was not possible to calculate an accurate sample size, and therefore, it was calculated based on a time period of one month. Second, presenting diagnosis and associated comorbidities were not included while collecting the data as that would have added to the significance of our analysis and results.

\section{Conclusions}

To conclude, poly-pharmacy is associated with a higher frequency of potential DDIs. Patients in developing countries are prescribed a more significant number of regular medications than in developed countries. As a result, they are more exposed to ADEs. Moreover, elderly patients are likely to be prescribed a higher number of regular-use drugs than the younger population.

There is a need for an efficient and cost-effective mechanism for preventing DDIs in low-income countries. This can be achieved by incorporating technology into prescription practice and by involving pharmacists in reviewing the medications for admitted patients. These steps have been shown to effectively reduce DDIs in developed countries.

\section{Additional Information \\ Disclosures}

Human subjects: Consent was obtained or waived by all participants in this study. Ethical Review Board issued approval PRM/21/14376. Animal subjects: All authors have confirmed that this study did not involve animal subjects or tissue. Conflicts of interest: In compliance with the ICMJE uniform disclosure form, all authors declare the following: Payment/services info: All authors have declared that no financial support was received from any organization for the submitted work. Financial relationships: All authors have declared that they have no financial relationships at present or within the previous three years with any organizations that might have an interest in the submitted work. Other relationships: All authors have declared that there are no other relationships or activities that could appear to have influenced the submitted work.

\section{References}

1. To err is human: building a safer health system . (2000). Accessed: August 20, 2018: https://en.wikipedia.org/wiki/To_Err_Is_Human_(report).

2. Budnitz DS, Lovegrove MC, Shehab N, Richards CL: Emergency hospitalizations for adverse drug events in older Americans. N Engl J Med. 2011, 365:2002-12. 10.1056/NEJMsa1103053 
3. Bates DW, Cullen DJ, Laird N, et al.: incidence of adverse drug events and potential adverse drug events. Implications for prevention. ADE Prevention Study Group. JAMA. 1995, 274:29-34. 10.1001/jama.1995.03530010043033

4. Bailey C, Peddie D, Wickham ME, et al.: Adverse drug event reporting systems: a systematic review. Br J Clin Pharmacol. 2016, 82:17-29. 10.1111/bcp.12944

5. Janković SM, Pejčić AV, Milosavljević MN, Opančina VD, Pešić NV, Nedeljković TT, Babić GM: Risk factors for potential drug-drug interactions in intensive care unit patients. J Crit Care. 2018, 43:1-6. 10.1016/j.jcrc.2017.08.021

6. Halkin H, Katzir I, Kurman I, Jan J, Malkin BB: Preventing drug interactions by online prescription screening in community pharmacies and medical practices. Clin Pharmacol Ther. 2001, 69:260-5. 10.1067/mcp.2001.114228

7. Garg AX, Adhikari NK, McDonald H, et al.: Effects of computerized clinical decision support systems on practitioner performance and patient outcomes: a systematic review. JAMA. 2005, 293:1223-38. 10.1001/jama.293.10.1223

8. Mekdad SS, Alsayed AA: Quality improvement project to reduce drug-related problems (DRPs) and potentially inappropriate medications (PIMs) in geriatrics cardiac clinic in Saudi Arabia. Can Geriatr J. 2019, 22:49-54. 10.5770/cgj.22.338

9. Weeks WB, Mills PD, Dittus RS, Aron DC, Batalden PB: Using an improvement model to reduce adverse drug events in VA facilities. Jt Comm J Qual Improv. 2001, 27:243-54. 10.1016/s1070-324127021-7

10. Mousavi S, Ghanbari G: Potential drug-drug interactions among hospitalized patients in a developing country. Caspian J Intern Med. 2017, 8:282-8. 10.22088/cjim.8.4.282

11. Ismail M, Iqbal Z, Khattak MB, et al.: Potential drug-drug interactions in internal medicine wards in hospital setting in Pakistan. Int J Clin Pharm. 2013, 35:455-62. 10.1007/s11096-013-9764-1

12. Bethi Y, Shewade DG, Dutta TK, Gitanjali B: Prevalence and predictors of potential drug-drug interactions in patients of internal medicine wards of a tertiary care hospital in India. Eur J Hosp Pharm. 2018, 25:317-21. 10.1136/ejhpharm-2017-001272

13. Sharifi H, Hasanloei MA, Mahmoudi J: Polypharmacy-induced drug-drug interactions; threats to patient safety. Drug Res (Stuttg). 2014, 64:633-7. 10.1055/s-0033-1363965

14. Tesfaye ZT, Nedi T: Potential drug-drug interactions in inpatients treated at the Internal Medicine ward of Tikur Anbessa Specialized Hospital. Drug Healthc Patient Saf. 2017, 9:71-6. 10.2147/DHPS.S126336

15. Straubhaar B, Krähenbühl S, Schlienger RG: The prevalence of potential drug-drug interactions in patients with heart failure at hospital discharge. Drug Saf. 2006, 29:79-90. 10.2165/00002018-200629010-00006

16. Pirmohamed M, James S, Meakin S, et al.: Adverse drug reactions as cause of admission to hospital: prospective analysis of 18820 patients. BMJ. 2004, 329:15-9. 10.1136/bmj.329.7456.15

17. Patel VK, Acharya LD, Rajakannan T, Surulivelrajan M, Guddattu V, Padmakumar R: Potential drug interactions in patients admitted to cardiology wards of a south Indian teaching hospital. Australas Med J. 2011, 4:9-14. 10.4066/AMJ.2011.450

18. Björkman IK, Fastbom J, Schmidt IK, Bernsten CB: Drug-drug interactions in the elderly. Ann Pharmacother. 2002, 36:1675-81. 10.1345/aph.1A484

19. Costa AJ: Potential drug interactions in an ambulatory geriatric population. Fam Pract. 1991, 8:234-6. 10.1093/fampra/8.3.234

20. Bhagavathula AS, Berhanie A, Tigistu H, Abraham Y, Getachew Y, Khan TM, Unakal C: Prevalence of potential drug-drug interactions among internal medicine ward in University of Gondar Teaching Hospital, Ethiopia. Asian Pac J Trop Biomed. 2014, 4:S204-8. 10.12980/APJTB.4.2014C1172

21. Egger SS, Drewe J, Schlienger RG: Potential drug-drug interactions in the medication of medical patients at hospital discharge. Eur J Clin Pharmacol. 2003, 58:773-8. 10.1007/s00228-002-0557-z

22. Riechelmann RP, Moreira F, Smaletz O, Saad ED: Potential for drug interactions in hospitalized cancer patients. Cancer Chemother Pharmacol. 2005, 56:286-90. 10.1007/s00280-004-0998-4

23. Johnell K, Klarin I: The relationship between number of drugs and potential drug-drug interactions in the elderly: a study of over 600,000 elderly patients from the Swedish Prescribed Drug Register. Drug Saf. 2007, 30:911-8. 10.2165/00002018-200730100-00009

24. Masnoon N, Shakib S, Kalisch-Ellett L, Caughey GE: What is polypharmacy? A systematic review of definitions. BMC Geriatr. 2017, 17:230. 10.1186/s12877-017-0621-2

25. Castilho EC, Reis AM, Borges TL, Siqueira LD, Miasso AI: Potential drug-drug interactions and polypharmacy in institutionalized elderly patients in a public hospital in Brazil. J Psychiatr Ment Health Nurs. 2018, 25:3-13. 10.1111/jpm.12431

26. Reeve E, Gnjidic D, Long J, Hilmer S: A systematic review of the emerging definition of 'deprescribing' with network analysis: implications for future research and clinical practice. Br J Clin Pharmacol. 2015, 80:125468. 10.1111/bcp.12732

27. Duncan P, Duerden M, Payne RA: Deprescribing: a primary care perspective . Eur J Hosp Pharm. 2017, 24:3742. 10.1136/ejhpharm-2016-000967

28. Reeve E, Andrews JM, Wiese MD, Hendrix I, Roberts MS, Shakib S: Feasibility of a patient-centered deprescribing process to reduce inappropriate use of proton pump inhibitors. Ann Pharmacother. 2015, 49:29-38. 10.1177/1060028014558290

29. Moorhouse P, Mallery LH: Palliative and therapeutic harmonization: a model for appropriate decisionmaking in frail older adults. J Am Geriatr Soc. 2012, 60:2326-32. 10.1111/j.1532-5415.2012.04210.x

30. Mangin D, Bahat G, Golomb BA, et al.: International group for reducing inappropriate medication use and polypharmacy (IGRIMUP): position statement and 10 recommendations for action. Drugs Aging. 2018, 35:575-87. 10.1007/s40266-018-0554-2 